\title{
Iteration-complexity analysis of a generalized alternating direction method of multipliers
}

\author{
V.A. Adona * M.L.N. Gonçalves * J.G. Melo *
}

May 18, 2017

\begin{abstract}
This paper analyzes the iteration-complexity of a generalized alternating direction method of multipliers (G-ADMM) for solving linearly constrained convex problems. This ADMM variant, which was first proposed by Bertsekas and Eckstein, introduces a relaxation parameter $\alpha \in(0,2)$ into the second ADMM subproblem. Our approach is to show that the G-ADMM is an instance of a hybrid proximal extragradient framework with some special properties, and, as a by product, we obtain ergodic iteration-complexity for the G-ADMM with $\alpha \in(0,2]$, improving and complementing related results in the literature. Additionally, we also present pointwise iteration-complexity for the G-ADMM.
\end{abstract}

2000 Mathematics Subject Classification: 47H05, 49M27, 90C25, 90C60, 65K10.

Key words: generalized alternating direction method of multipliers, hybrid extragradient method, convex program, pointwise iteration-complexity, ergodic iteration-complexity.

\section{Introduction}

This paper considers the following linearly constrained convex optimization problem

$$
\min \left\{f(x)+g(y): A x+B y=b, x \in \mathbb{R}^{n}, y \in \mathbb{R}^{p}\right\}
$$

where $f: \mathbb{R}^{n} \rightarrow \mathbb{R}$ and $g: \mathbb{R}^{p} \rightarrow \mathbb{R}$ are convex functions, $A \in \mathbb{R}^{m \times n}, B \in \mathbb{R}^{m \times p}$ and $b \in \mathbb{R}^{m}$. Problems with separable structure such as (1) arises in many applications areas, for instance, machine learning, compressive sensing and image processing. One popular method for solving (1), taking advantages of its special structure, is the alternating direction method of multipliers (ADMM) [13, 15]; for detailed reviews, see [2, 14. Many variants of it have been considered in the literature; see, for example, $15,8,110,12,18,19,20,21$, 23, 27. The ADMM variant studied here is the generalized ADMM [1] (G-ADMM) with proximal terms, described as follows: given $\left(x_{k-1}, y_{k-1}, \gamma_{k-1}\right)$ compute $\left(x_{k}, y_{k}, \gamma_{k}\right)$ as

$$
\begin{aligned}
& x_{k} \in \operatorname{argmin}_{x}\left\{f(x)-\left\langle\gamma_{k-1}, A x\right\rangle+\frac{\beta}{2}\left\|A x+B y_{k-1}-b\right\|^{2}+\frac{1}{2}\left\|x-x_{k-1}\right\|_{H_{1}}^{2}\right\}, \\
& y_{k} \in \operatorname{argmin}_{y}\left\{g(y)-\left\langle\gamma_{k-1}, B y\right\rangle+\frac{\beta}{2}\left\|\alpha\left(A x_{k}+B y_{k-1}-b\right)+B\left(y-y_{k-1}\right)\right\|^{2}+\frac{1}{2}\left\|y-y_{k-1}\right\|_{H_{2}}^{2}\right\}, \\
& \gamma_{k}=\gamma_{k-1}-\beta\left[\alpha\left(A x_{k}+B y_{k-1}-b\right)+B\left(y_{k}-y_{k-1}\right)\right]
\end{aligned}
$$

where $\beta>0$ is a fixed penalty parameter, $\left(H_{1}, H_{2}\right) \in \mathbb{R}^{n \times n} \times \mathbb{R}^{p \times p}$ are symmetric and positive semi-definite matrices, $\alpha \in(0,2]$ is a relaxation factor and $\|\cdot\|_{H_{i}}^{2}:=\left\langle H_{i}(\cdot), \cdot\right\rangle, i=1,2$. Different ADMM variants studied

${ }^{*}$ Institute of Mathematics and Statistics, Federal University of Goias, Campus II- Caixa Postal 131, CEP 74001-970, GoiâniaGO, Brazil. (E-mails: vandomat32@hotmail.com, maxlng@ufg.br and jefferson@ufg.br). The work of these authors was supported in part by CNPq Grant 444134/2014-0, 309370/2014-0 and FAPEG/GO. 
in the literature can be seen as particular instances of the G-ADMM by appropriately choosing the matrices $H_{i}(i=1,2)$ and the relaxation parameter $\alpha$. By setting $\left(H_{1}, H_{2}\right)=(0,0)$ and $\alpha=1$, the G-ADMM reduces to the standard ADMM. The use of over-relaxation parameter $(\alpha>1)$ in some applications can accelerate the standard ADMM; see, for instance, [1, 9]. By choosing $\left(H_{1}, H_{2}\right)=\left(\tau_{1} I_{n}-\beta A^{*} A, \tau_{2} I_{p}-\beta B^{*} B\right)$ for some $\tau_{1} \geq \beta\|A\|^{2}, \tau_{2} \geq \beta\|B\|^{2}$ (* stands for the adjoint operator), the G-ADMM subproblems may become much easier to solve, since the quadratic terms involving $A^{*} A$ and $B^{*} B$ vanish; see, for example, [8, 32, 33, for discussion. It is well-known that an optimal solution $\left(x^{*}, y^{*}\right)$ for problem (11) can be obtained by finding a solution $\left(x^{*}, y^{*}, \gamma^{*}\right)$ of the following Lagrangian system

$$
0 \in \partial f(x)-A^{*} \gamma, \quad 0 \in \partial g(y)-B^{*} \gamma, \quad A x+B y-b=0,
$$

where $\gamma^{*}$ is an associated Lagrange multiplier.

In this paper, we are interested in analyzing iteration-complexity of the G-ADMM to obtain an "approximate solution" of the Lagrangian system (3). Specifically, for a given tolerance $\varepsilon>0$, we show that in at most $\mathcal{O}(1 / \varepsilon)$ iterations of the G-ADMM, we obtain, in the ergodic sense, an " $\varepsilon$-approximate" solution $(\hat{x}, \hat{y}, \hat{\gamma})$ and a residual $\hat{v}=\left(\hat{v}_{1}, \hat{v}_{2}, \hat{v}_{3}\right)$ of (3) satisfying

$$
\hat{v}_{1} \in \partial_{\varepsilon_{1}} f(\hat{x})-A^{*} \hat{\gamma}, \quad \hat{v}_{2} \in \partial_{\varepsilon_{2}} g(\hat{y})-B^{*} \hat{\gamma}, \quad \hat{v}_{3}=A \hat{x}+B \hat{y}-b, \quad\|\hat{v}\|_{\left(H_{1}, H_{2}\right)} \leq \varepsilon, \quad \varepsilon_{1}+\varepsilon_{2} \leq \varepsilon,
$$

where the symbol $\partial_{\varepsilon}$ stands for $\varepsilon$-subdiferential, and $\|\cdot\|_{\left(H_{1}, H_{2}\right)}$ is a norm (seminorm) depending on the matrices $H_{1}$ and $H_{2}$. Our approach is to show that the G-ADMM is an instance of a hybrid proximal extragradient (HPE) framework (see 24, 29]) with a very special property, namely, a key parameter sequence $\left\{\rho_{k}\right\}$ associated to the sequence generated by the method is upper bounded by a multiple of $d_{0}$ (a parameter measuring, in some sense, the distance of the initial point to the solution set). This result is essential to obtain the ergodic iteration-complexity of the G-ADMM with relaxation parameter $\alpha \in(0,2]$. Additionally, we also present pointwise iteration-complexity for the G-ADMM with $\alpha \in(0,2)$.

Convergence rates of the G-ADMM and related variants have been studied by many authors in different contexts. In 12, the authors obtain pointwise and ergodic convergence rate bounds for the G-ADMM with $\alpha \in(0,2)$. Paper [26] studies linear convergence of the G-ADMM under additional assumptions. Some strategies are also proposed in order to choose the relaxation and penalty parameters. Linear convergence of the G-ADMM is also studied in [31] on a general setting. Paper [30 studies the G-ADMM as a particular case of a general scheme in a Hilbert space and measures, in an ergodic sense, a "partial" primal-dual gap associated to the augmented Lagrangian of problem (1). Paper [6] studies convergence rates of a generalized proximal point algorithm and obtains, as a by product, convergence rates of the particular instance of the G-ADMM in which $\left(H_{1}, H_{2}\right)=(0,0)$. It is worth mentioning that the previous ergodic convergence results for the G-ADMM are not focused in solving (3) approximately in the sense of our paper. Iteration-complexity study of the standard ADMM and some variants in the setting of the HPE framework have been considered in [16, 18, 25. Finally, convergence rates of ADMM variants using a different approach have been studied in 7, 8, 18, 20, 21, 22, 23, 26, 27, to name just a few.

Organization of the paper. Section 2 is divided into two subsections, Subsection 2.1 presents our notation and basic results. Subsection 2.2 is devoted to the study of a modified HPE framework and present its main iteration-complexity results whose proofs are given in Section $\mathrm{A}$. Section 3 is divided into three subsections. Subsection 3.1 formally describes the generalized ADMM and Subsection 3.2 contains some auxiliary results. The pointwise and ergodic iteration-complexity results for the G-ADMM are given in Subsection 3.3 .

\section{Preliminary results}

This section is divided into two subsections: The first one presents our notation and basic results, and the second one describes a modified HPE framework and present its iteration-complexity bounds. 


\subsection{Notation and basic definitions}

This subsection presents some definitions, notation and basic results used in this paper.

Let $\mathcal{V}$ be a finite-dimensional real vector space with inner product and associated norm denoted by $\langle\cdot, \cdot\rangle$ and $\|\cdot\|$, respectively. For a given self-adjoint positive semidefinite linear operator $Q: \mathcal{V} \rightarrow \mathcal{V}$, the seminorm induced by $Q$ on $\mathcal{V}$ is defined by $\|\cdot\|_{Q}=\langle Q(\cdot), \cdot\rangle^{1 / 2}$. Since $\langle Q(\cdot), \cdot\rangle$ is symmetric and bilinear, for all $v, \tilde{v} \in \mathcal{V}$, we have

$$
2\langle Q v, \tilde{v}\rangle \leq\|v\|_{Q}^{2}+\|\tilde{v}\|_{Q}^{2}
$$

Given a set-valued operator $T: \mathcal{V} \rightrightarrows \mathcal{V}$, its domain and graph are defined, respectively, as

$$
\operatorname{Dom} T:=\{v \in \mathcal{V}: T(v) \neq \emptyset\} \quad \text { and } \quad \operatorname{Gr}(T)=\{(v, \tilde{v}) \in \mathcal{V} \times \mathcal{V} \mid \tilde{v} \in T(v)\} .
$$

The operator $T$ is said to be monotone if

$$
\langle u-v, \tilde{u}-\tilde{v}\rangle \geq 0 \quad \forall(u, \tilde{u}),(v, \tilde{v}) \in \operatorname{Gr}(T) .
$$

Moreover, $T$ is maximal monotone if it is monotone and there is no other monotone operator $S$ such that $\operatorname{Gr}(T) \subset \operatorname{Gr}(S)$. Given a scalar $\varepsilon \geq 0$, the $\varepsilon$-enlargement $T^{[\varepsilon]}: \mathcal{V} \rightrightarrows \mathcal{V}$ of a monotone operator $T: \mathcal{V} \rightrightarrows \mathcal{V}$ is defined as

$$
T^{[\varepsilon]}(v):=\{\tilde{v} \in \mathcal{V}:\langle\tilde{v}-\tilde{u}, v-u\rangle \geq-\varepsilon, \quad \forall(u, \tilde{u}) \in G r(T)\} \quad \forall v \in \mathcal{V} .
$$

The $\varepsilon$-subdifferential of a proper closed convex function $f: \mathcal{V} \rightarrow[-\infty, \infty]$ is defined by

$$
\partial_{\varepsilon} f(v):=\{u \in \mathcal{V}: f(\tilde{v}) \geq f(v)+\langle u, \tilde{v}-v\rangle-\varepsilon, \forall \tilde{v} \in \mathcal{V}\} \quad \forall v \in \mathcal{V} .
$$

When $\varepsilon=0$, then $\partial_{0} f(v)$ is denoted by $\partial f(v)$ and is called the subdifferential of $f$ at $v$. It is well known that the subdifferential operator of a proper closed convex function is maximal monotone [28].

The next theorem is a consequence of the transportation formula in [4, Theorem 2.3] combined with [3, Proposition 2(i)].

Theorem 2.1. Suppose $T: \mathcal{V} \rightrightarrows \mathcal{V}$ is maximal monotone and let $\tilde{v}_{i}, v_{i} \in \mathcal{V}$, for $i=1, \cdots, k$, be such that $v_{i} \in T\left(\tilde{v}_{i}\right)$ and define

$$
\tilde{v}_{k}^{a}=\frac{1}{k} \sum_{i=1}^{k} \tilde{v}_{i}, \quad v_{k}^{a}=\frac{1}{k} \sum_{i=1}^{k} v_{i}, \quad \varepsilon_{k}^{a}=\frac{1}{k} \sum_{i=1}^{k}\left\langle v_{i}, \tilde{v}_{i}-\tilde{v}_{k}^{a}\right\rangle .
$$

Then, the following hold:

(a) $\varepsilon_{k}^{a} \geq 0$ and $v_{k}^{a} \in T^{\left[\varepsilon_{k}^{a}\right]}\left(\tilde{v}_{k}^{a}\right)$;

(b) if, in addition, $T=\partial f$ for some proper closed and convex function $f$, then $v_{k}^{a} \in \partial_{\varepsilon_{k}^{a}} f\left(\tilde{v}_{k}^{a}\right)$.

\subsection{A HPE-type framework}

This subsection describes the modified HPE framework and its corresponding pointwise and ergodic iterationcomplexity bounds.

Let $\mathcal{Z}$ be a finite-dimensional real vector space with inner product and induced norm denoted by $\langle\cdot, \cdot\rangle$ and $\|\cdot\|=\sqrt{\langle\cdot, \cdot\rangle}$, respectively. Our problem of interest in this section is the monotone inclusion problem (MIP)

$$
0 \in T(z)
$$

where $T: \mathcal{Z} \rightrightarrows \mathcal{Z}$ is a maximal monotone operator. We assume that the solution set of (6), denoted by $T^{-1}(0)$, is nonempty.

We now state the modified HPE framework for solving (6). 


\section{A modified HPE framework for solving (6).}

(0) Let $z_{0} \in \mathcal{Z}, \eta_{0} \in \mathbb{R}_{+}, \sigma \in[0,1]$ and a self-adjoint positive semidefinite linear operator $M: \mathcal{Z} \rightarrow \mathcal{Z}$ be given, and set $k=1$;

(1) obtain $\left(z_{k}, \tilde{z}_{k}, \eta_{k}\right) \in \mathcal{Z} \times \mathcal{Z} \times \mathbb{R}_{+}$such that

$$
\begin{aligned}
M\left(z_{k-1}-z_{k}\right) & \in T\left(\tilde{z}_{k}\right) \\
\left\|\tilde{z}_{k}-z_{k}\right\|_{M}^{2}+\eta_{k} & \leq \sigma\left\|\tilde{z}_{k}-z_{k-1}\right\|_{M}^{2}+\eta_{k-1}
\end{aligned}
$$

(2) set $k \leftarrow k+1$ and go to step 1 .

end

Some remarks about the modified HPE framework are in order. First, it is an instance of the non-Euclidean HPE framework of [17] with $\lambda_{k}=1, \varepsilon_{k}=0$ and $(d w)_{z}\left(z^{\prime}\right)=(1 / 2)\left\|z-z^{\prime}\right\|_{M}^{2}, \forall z, z^{\prime} \in \mathcal{Z}$. Second, the way to obtain $\left(z_{k}, \tilde{z}_{k}, \eta_{k}\right)$ will depend on the particular instance of the framework and properties of the operator $T$. In section 3.2 we will show that a generalized ADMM can be seen as an instance of the HPE framework specifying, in particular, how this triple $\left(z_{k}, \tilde{z}_{k}, \eta_{k}\right)$ can be obtained. Third, if $M$ is positive definite and $\sigma=\eta_{0}=0$, then (8) implies that $\eta_{k}=0$ and $z_{k}=\tilde{z}_{k}$ for every $k$, and hence that $M\left(z_{k-1}-z_{k}\right) \in T\left(z_{k}\right)$ in view of (7). Therefore, the HPE error conditions (7)-(8) can be viewed as a relaxation of an iteration of the exact proximal point method.

In the following, we present pointwise and ergodic iteration-complexity results for the modified HPE framework. Let $d_{0}$ be the distance of $z_{0}$ to the solution set of $T^{-1}(0)$, i.e.,

$$
d_{0}=\inf \left\{\left\|z^{*}-z_{0}\right\|_{M}^{2}: z^{*} \in T^{-1}(0)\right\} .
$$

For convenience of the reader and completeness, the proof of the next two results are presented in Appendix $\mathrm{A}$

Theorem 2.2. (Pointwise convergence of the HPE) Consider the sequence $\left\{\left(z_{k}, \tilde{z}_{k}, \eta_{k}\right)\right\}$ generated by the modified HPE framework with $\sigma<1$. Then, for every $k \geq 1$, there hold $0 \in M\left(z_{k}-z_{k-1}\right)+T\left(\tilde{z}_{k}\right)$ and there exists $i \leq k$ such that

$$
\left\|z_{i}-z_{i-1}\right\|_{M} \leq \frac{1}{\sqrt{k}} \sqrt{\frac{2(1+\sigma) d_{0}+4 \eta_{0}}{1-\sigma}}
$$

where $d_{0}$ is as defined in (9).

Next, we present the ergodic convergence of the modified HPE framework. Before, let us consider the following ergodic sequences

$$
\tilde{z}_{k}^{a}=\frac{1}{k} \sum_{i=1}^{k} \tilde{z}_{i}, \quad r_{k}^{a}=\frac{1}{k} \sum_{i=1}^{k}\left(z_{i}-z_{i-1}\right), \quad \varepsilon_{k}^{a}:=\frac{1}{k} \sum_{i=1}^{k}\left\langle M\left(z_{i}-z_{i-1}\right), \tilde{z}_{k}^{a}-\tilde{z}_{i}\right\rangle, \quad \forall k \geq 1 .
$$

Theorem 2.3. (Ergodic convergence of the HPE) Consider the ergodic sequence $\left\{\left(\tilde{z}_{k}^{a}, r_{k}^{a}, \varepsilon_{k}^{a}\right)\right\}$ as in (10). For every $k \geq 1$, there hold $\varepsilon_{k}^{a} \geq 0,0 \in M r_{k}^{a}+T^{\left[\varepsilon_{k}^{a}\right]}\left(\tilde{z}_{k}^{a}\right)$ and

$$
\left\|r_{k}^{a}\right\|_{M} \leq \frac{2 \sqrt{d_{0}+\eta_{0}}}{k}, \quad \varepsilon_{k}^{a} \leq \frac{3\left[3\left(d_{0}+\eta_{0}\right)+\sigma \rho_{k}\right]}{2 k},
$$

where

$$
\rho_{k}:=\max _{i=1, \ldots, k}\left\|\tilde{z}_{i}-z_{i-1}\right\|_{M}^{2},
$$

and $d_{0}$ is as defined in (91). Moreover, the sequence $\left\{\rho_{k}\right\}$ is bounded under either one of the following situations:

(a) $\sigma<1$, in which case

$$
\rho_{k} \leq \frac{d_{0}+\eta_{0}}{1-\sigma}
$$


(b) Dom $T$ is bounded, in which case

$$
\rho_{k} \leq 2\left[d_{0}+\eta_{0}+D\right]
$$

where $D:=\sup \left\{\left\|y^{\prime}-y\right\|_{M}^{2}: y, y^{\prime} \in \operatorname{Dom} T\right\}$.

If $\sigma<1$ or $\operatorname{Dom} T$ is bounded, it follows from Theorem 2.3 that $\left\{\rho_{k}\right\}$ is bounded and hence $\max \left\{\left\|r_{k}^{a}\right\|_{M}, \varepsilon_{k}^{a}\right\}=$ $\mathcal{O}(1 / k)$. However, it may happen that the sequence $\left\{\rho_{k}\right\}$ is bounded even when $\sigma=1$. Indeed, in the next section, we will present a generalized ADMM which is an instance of the modified HPE framework satisfying this case (see Lemma (3.5)).

\section{The generalized $\mathrm{ADMM}$ and its convergence rates}

The main goal of this section is to describe the generalized ADMM for solving (1) and present pointwise and ergodic iteration-complexity results for it. Our iteration-complexity bounds are obtained by showing that this ADMM variant is a special case of the modified HPE framework of Section 2.2 .

Throughout this section, we assume that:

A1) the problem (11) has an optimal solution $\left(x^{*}, y^{*}\right)$ and an associated Lagrange multiplier $\gamma^{*}$, or equivalently, the inclusion

$$
0 \in T(x, y, \gamma):=\left[\begin{array}{l}
\partial f(x)-A^{*} \gamma \\
\partial g(y)-B^{*} \gamma \\
A x+B y-b
\end{array}\right]
$$

has a solution $\left(x^{*}, y^{*}, \gamma^{*}\right)$;

\subsection{The generalized ADMM}

In this subsection, we recall the generalized ADMM first proposed by Eckstein and Bertsekas (see [9, 11, 12]) for solving (11).

\section{Generalized ADMM}

(0) Let an initial point $\left(x_{0}, y_{0}, \gamma_{0}\right) \in \mathbb{R}^{n} \times \mathbb{R}^{p} \times \mathbb{R}^{m}$, a penalty parameter $\beta>0$, a relaxation factor $\alpha \in(0,2]$, and symmetric positive semidefinite matrices $H_{1} \in \mathbb{R}^{n \times n}$ and $H_{2} \in \mathbb{R}^{p \times p}$ be given, and set $k=1$;

(1) compute an optimal solution $x_{k} \in \mathbb{R}^{n}$ of the subproblem

$$
\min _{x \in \mathbb{R}^{n}}\left\{f(x)-\left\langle\gamma_{k-1}, A x\right\rangle+\frac{\beta}{2}\left\|A x+B y_{k-1}-b\right\|^{2}+\frac{1}{2}\left\|x-x_{k-1}\right\|_{H_{1}}^{2}\right\}
$$

and compute an optimal solution $y_{k} \in \mathbb{R}^{p}$ of the subproblem

$$
\min _{y \in \mathbb{R}^{p}}\left\{g(y)-\left\langle\gamma_{k-1}, B y\right\rangle+\frac{\beta}{2}\left\|\alpha\left(A x_{k}+B y_{k-1}-b\right)+B\left(y-y_{k-1}\right)\right\|^{2}+\frac{1}{2}\left\|y-y_{k-1}\right\|_{H_{2}}^{2}\right\} ;
$$

(2) set

$$
\gamma_{k}=\gamma_{k-1}-\beta\left[\alpha\left(A x_{k}+B y_{k-1}-b\right)+B\left(y_{k}-y_{k-1}\right)\right]
$$

and $k \leftarrow k+1$, and go to step (1).

end

The generalized ADMM has different features depending on the choices of the operators $H_{1}, H_{2}$, and the relaxation factor $\alpha$. For instance, by taking $\alpha=1$ and $\left(H_{1}, H_{2}\right)=(0,0)$, it reduces to the standard ADMM, and $\alpha=1$ and $\left(H_{1}, H_{2}\right)=\left(\tau_{1} I_{n}-\beta A^{*} A, \tau_{2} I_{p}-\beta B^{*} B\right)$ with $\tau_{1}>\beta\left\|A^{*} A\right\|$ and $\tau_{2}>\beta\left\|B^{*} B\right\|$, it reduces to 
the linearized ADMM. The latter method basically consists of canceling the quadratic terms $(\beta / 2)\|A x\|^{2}$ and $(\beta / 2)\|B y\|^{2}$ in (14) and (15), respectively. More specifically, the subproblems (14) and (15) become

$$
\begin{gathered}
\min _{x \in \mathbb{R}^{n}}\left\{f(x)-\left\langle\gamma_{k-1}-\beta\left(A x_{k-1}+B y_{k-1}-b\right), A x\right\rangle+\frac{\tau_{1}}{2}\left\|x-x_{k-1}\right\|^{2}\right\}, \\
\min _{y \in \mathbb{R}^{p}}\left\{g(y)-\left\langle\gamma_{k-1}-\alpha \beta\left(A x_{k}+B y_{k-1}-b\right), B y\right\rangle+\frac{\tau_{2}}{2}\left\|y-y_{k-1}\right\|^{2}\right\} .
\end{gathered}
$$

In many applications, the above subproblems are much easier to solve or even have closed-form solutions (see [21, 32, 33 for more details). We also mention that depending on the structure of problem (11), other choices of $H_{1}$ and $H_{2}$ may be recommended; see, for instance, 8 . (although the latter reference considers $\alpha=1$, it is clear that the same discussion regarding the choices of $H_{1}$ and $H_{2}$ holds for arbitrary $\left.\alpha \in(0,2)\right)$. The generalized ADMM with over-relaxation parameter $(\alpha>1)$ may present computational advantages over the standard ADMM (see, for example, 9]).

\subsection{The generalized ADMM as an instance of the modified HPE framework}

Our aim in this subsection is to show that the generalized ADMM is an instance of the modified HPE framework for solving the inclusion problem (13) and, as a by-product, pointwise and ergodic iteration-complexity bounds results for the generalized ADMM will be presented in Subsection 3.3 .

Let us first introduce the elements required by the setting of Subsection 2.2 Consider the vector space $\mathcal{Z}:=\mathbb{R}^{n} \times \mathbb{R}^{p} \times \mathbb{R}^{m}$, the linear operator

$$
M:=\left[\begin{array}{ccc}
H_{1} & 0 & 0 \\
0 & \left(H_{2}+\frac{\beta}{\alpha} B^{*} B\right) & \frac{(1-\alpha)}{\alpha} B^{*} \\
0 & \frac{(1-\alpha)}{\alpha} B & \frac{1}{\alpha \beta} I_{m}
\end{array}\right],
$$

and the quantity

$$
d_{0}:=\inf _{(x, y, \gamma) \in T^{-1}(0)}\left\{\left\|\left(x-x_{0}, y-y_{0}, \gamma-\gamma_{0}\right)\right\|_{M}^{2}\right\} .
$$

It is easy to verify that $M$ is a symmetric positive semidefinite matrix for every $\beta>0$ and $\alpha \in(0,2]$. Let $\left\{\left(x_{k}, y_{k}, \gamma_{k}\right)\right\}$ be the sequence generated by the generalized ADMM. In order to simplify some relations in the results below, define the sequence $\left\{\left(\Delta x_{k}, \Delta y_{k}, \Delta \gamma_{k}, \tilde{\gamma}_{k}\right)\right\}$ as

$$
\Delta x_{k}=x_{k}-x_{k-1}, \quad \Delta y_{k}=y_{k}-y_{k-1}, \quad \Delta \gamma_{k}=\gamma_{k}-\gamma_{k-1}, \quad \tilde{\gamma}_{k}=\gamma_{k-1}-\beta\left(A x_{k}+B y_{k-1}-b\right)
$$

for every $k \geq 1$.

We next present two technical results on the generalized ADMM.

Lemma 3.1. Let $\left\{\left(x_{k}, y_{k}, \gamma_{k}\right)\right\}$ be generated by the generalized ADMM and consider $\left\{\left(\Delta x_{k}, \Delta y_{k}, \Delta \gamma_{k}, \tilde{\gamma}_{k}\right)\right\}$ as in (19). Then, for every $k \geq 1$,

$$
\begin{aligned}
\tilde{\gamma}_{k}-\gamma_{k-1} & =\frac{1}{\alpha}\left[\Delta \gamma_{k}+\beta B \Delta y_{k}\right], \\
0 & \in H_{1} \Delta x_{k}+\left[\partial f\left(x_{k}\right)-A^{*} \tilde{\gamma}_{k}\right], \\
0 & \in\left(H_{2}+\frac{\beta}{\alpha} B^{*} B\right) \Delta y_{k}+\frac{(1-\alpha)}{\alpha} B^{*} \Delta \gamma_{k}+\left[\partial g\left(y_{k}\right)-B^{*} \tilde{\gamma}_{k}\right], \\
0 & =\frac{(1-\alpha)}{\alpha} B \Delta y_{k}+\frac{1}{\alpha \beta} \Delta \gamma_{k}+\left[A x_{k}+B y_{k}-b\right] .
\end{aligned}
$$

As a consequence, $z_{k}:=\left(x_{k}, y_{k}, \gamma_{k}\right)$ and $\tilde{z}_{k}:=\left(x_{k}, y_{k}, \tilde{\gamma}_{k}\right)$ satisfy the inclusion (7) with $T$ and $M$ as in (13) and (17), respectively. 
Proof. It follows from definitions of $\gamma_{k}$ and $\tilde{\gamma}_{k}$ in (16) and (19), respectively, that

$$
\frac{1}{\alpha}\left(\gamma_{k}-\gamma_{k-1}\right)+\frac{\beta}{\alpha} B\left(y_{k}-y_{k-1}\right)=-\beta\left(A x_{k}+B y_{k-1}-b\right)=\tilde{\gamma}_{k}-\gamma_{k-1},
$$

which, combined with definitions of $\Delta y_{k}$ and $\Delta \gamma_{k}$ in (19), proves (20). From the optimality condition for (14), we have

$$
0 \in \partial f\left(x_{k}\right)-A^{*}\left(\gamma_{k-1}-\beta\left(A x_{k}+B y_{k-1}-b\right)\right)+H_{1}\left(x_{k}-x_{k-1}\right),
$$

which, combined with definitions of $\tilde{\gamma}_{k}$ and $\Delta x_{k}$ in (19), yields (21). Similarly, from the optimality condition for (15) and definitions of $\gamma_{k}$ and $\Delta y_{k}$ in (16) and (21), respectively, we obtain

$$
\begin{aligned}
0 & \in \partial g\left(y_{k}\right)-B^{*}\left[\gamma_{k-1}-\beta \alpha\left(A x_{k}+B y_{k-1}-b\right)+\beta B\left(y_{k}-y_{k-1}\right)\right]+H_{2}\left(y_{k}-y_{k-1}\right) \\
& =\partial g\left(y_{k}\right)-B^{*} \gamma_{k}+H_{2} \Delta y_{k} .
\end{aligned}
$$

On the other hand, note that (20) implies that

$$
\gamma_{k}=\tilde{\gamma}_{k}+\left(\gamma_{k}-\gamma_{k-1}\right)-\left(\tilde{\gamma}_{k}-\gamma_{k-1}\right)=\tilde{\gamma}_{k}-\frac{(1-\alpha)}{\alpha} \Delta \gamma_{k}-\frac{\beta}{\alpha} B \Delta y_{k},
$$

which in turn, combined with (24), gives (22). The relation (23) follows immediately from (16).

Now, the last statement of the lemma follows directly by (21)-(23) and definitions of $T$ and $M$ given in (13) and (17), respectively.

Lemma 3.2. The sequences $\left\{\Delta y_{k}\right\}$ and $\left\{\Delta \gamma_{k}\right\}$ defined in (19) satisfy

$$
2\left\langle B \Delta y_{1}, \Delta \gamma_{1}\right\rangle \geq\left\|\Delta y_{1}\right\|_{H_{2}}^{2}-4 d_{0}, \quad 2\left\langle B \Delta y_{k}, \Delta \gamma_{k}\right\rangle \geq\left\|\Delta y_{k}\right\|_{H_{2}}^{2}-\left\|\Delta y_{k-1}\right\|_{H_{2}}^{2} \quad \forall k \geq 2,
$$

where $d_{0}$ is as in (18).

Proof. Let a point $z^{*}:=\left(x^{*}, y^{*}, \gamma^{*}\right)$ be such that $0 \in T\left(x^{*}, y^{*}, \gamma^{*}\right)$ (see assumption A1) and consider $z_{i}:=$ $\left(x_{i}, y_{i}, \gamma_{i}\right), i=0,1$. First, note that

$$
0 \leq \frac{\beta}{\alpha}\left\|B \Delta y_{1}\right\|^{2}+\frac{2}{\alpha}\left\langle B \Delta y_{1}, \Delta \gamma_{1}\right\rangle+\frac{1}{\alpha \beta}\left\|\Delta \gamma_{1}\right\|^{2},
$$

where $\Delta y_{1}$ and $\Delta \gamma_{1}$ are as in (19). Hence, by adding $\left\|\Delta y_{1}\right\|_{H_{2}}^{2}-2\left\langle B \Delta y_{1}, \Delta \gamma_{1}\right\rangle$ to both sides of the above inequality, we obtain

$$
\begin{aligned}
\left\|\Delta y_{1}\right\|_{H_{2}}^{2}-2\left\langle B \Delta y_{1}, \Delta \gamma_{1}\right\rangle & \leq\left\|\Delta y_{1}\right\|_{H_{2}}^{2}+\frac{\beta}{\alpha}\left\|B \Delta y_{1}\right\|^{2}+2 \frac{(1-\alpha)}{\alpha}\left\langle B \Delta y_{1}, \Delta \gamma_{1}\right\rangle+\frac{1}{\alpha \beta}\left\|\Delta \gamma_{1}\right\|^{2} \\
& \leq\left\|z_{1}-z_{0}\right\|_{M}^{2} \leq 2\left(\left\|z^{*}-z_{1}\right\|_{M}^{2}+\left\|z^{*}-z_{0}\right\|_{M}^{2}\right),
\end{aligned}
$$

where $M$ is as in (17) and the last inequality is a consequence of (4) with $Q=M$. On the other hand, taking $\tilde{z}_{1}=\left(x_{1}, y_{1}, \tilde{\gamma}_{1}\right)$, Lemma 3.1 implies that $\left(z_{0}, z_{1}, \tilde{z}_{1}\right)$ satisfies (7) with $T$ and $M$ as in (13) and (17), respectively; namely, $M\left(z_{0}-z_{1}\right) \in T\left(\tilde{z}_{1}\right)$. Hence, since $0 \in T\left(z^{*}\right)$ and $T$ is monotone, we obtain $\left\langle M\left(z_{0}-z_{1}\right), \tilde{z}_{1}-z^{*}\right\rangle \geq 0$. Thus, it follows that

$$
\begin{aligned}
\left\|z^{*}-z_{1}\right\|_{M}^{2}-\left\|z^{*}-z_{0}\right\|_{M}^{2} & =\left\|\left(z^{*}-\tilde{z}_{1}\right)+\left(\tilde{z}_{1}-z_{1}\right)\right\|_{M}^{2}-\left\|\left(z^{*}-\tilde{z}_{1}\right)+\left(\tilde{z}_{1}-z_{0}\right)\right\|_{M}^{2} \\
& =\left\|\tilde{z}_{1}-z_{1}\right\|_{M}^{2}+2\left\langle M\left(z_{0}-z_{1}\right), z^{*}-\tilde{z}_{1}\right\rangle-\left\|\tilde{z}_{1}-z_{0}\right\|_{M}^{2} \\
& \leq\left\|\tilde{z}_{1}-z_{1}\right\|_{M}^{2}-\left\|\tilde{z}_{1}-z_{0}\right\|_{M}^{2} .
\end{aligned}
$$

Combining (19) and (20), we have $\tilde{\gamma}_{1}-\gamma_{1}=\left[(1-\alpha) \Delta \gamma_{1}+\beta B \Delta y_{1}\right] / \alpha$. Hence, using the definitions of $M, z_{1}$ and $\tilde{z}_{1}$, we obtain

$$
\left\|\tilde{z}_{1}-z_{1}\right\|_{M}^{2}=\frac{1}{\alpha \beta}\left\|\tilde{\gamma}_{1}-\gamma_{1}\right\|^{2}=\frac{\beta}{\alpha^{3}}\left\|B \Delta y_{1}\right\|^{2}+2 \frac{(1-\alpha)}{\alpha^{3}}\left\langle B \Delta y_{1}, \Delta \gamma_{1}\right\rangle+\frac{(1-\alpha)^{2}}{\alpha^{3} \beta}\left\|\Delta \gamma_{1}\right\|^{2}
$$


and

$$
\begin{aligned}
\left\|\tilde{z}_{1}-z_{0}\right\|_{M}^{2} & \geq \frac{\beta}{\alpha}\left\|B\left(y_{1}-y_{0}\right)\right\|^{2}+\frac{2(1-\alpha)}{\alpha}\left\langle B\left(y_{1}-y_{0}\right), \tilde{\gamma}_{1}-\gamma_{0}\right\rangle+\frac{1}{\alpha \beta}\left\|\tilde{\gamma}_{1}-\gamma_{0}\right\|^{2} \\
& =\left(\frac{\beta}{\alpha}+2 \frac{(1-\alpha) \beta}{\alpha^{2}}+\frac{\beta}{\alpha^{3}}\right)\left\|B \Delta y_{1}\right\|^{2}+2\left(\frac{(1-\alpha)}{\alpha^{2}}+\frac{1}{\alpha^{3}}\right)\left\langle B \Delta y_{1}, \Delta \gamma_{1}\right\rangle+\frac{1}{\alpha^{3} \beta}\left\|\Delta \gamma_{1}\right\|^{2},
\end{aligned}
$$

where the last equality is due to (19) and (20). Hence, it is easy to see that

$$
\left\|\tilde{z}_{1}-z_{1}\right\|_{M}^{2}-\left\|\tilde{z}_{1}-z_{0}\right\|_{M}^{2} \leq \frac{(\alpha-2)}{\alpha^{2}}\left\|\sqrt{\beta} B \Delta y_{1}+\frac{1}{\sqrt{\beta}} \Delta \gamma_{1}\right\|^{2} \leq 0 .
$$

Thus, it follows from (27) that

$$
\left\|z^{*}-z_{1}\right\|_{M}^{2} \leq\left\|z^{*}-z_{0}\right\|_{M}^{2}
$$

which, combined with (26), yields

$$
\left\|\Delta y_{1}\right\|_{H_{2}}^{2}-2\left\langle B \Delta y_{1}, \Delta \gamma_{1}\right\rangle \leq 4\left\|z^{*}-z_{0}\right\|_{M}^{2} .
$$

Therefore, the first inequality in (25) follows from definition of $d_{0}$ (see (18)) and the fact that $z^{*} \in T^{-1}(0)$ is arbitrary.

Let us now prove the second inequality in (25). First, from the optimality condition of (15) and (16), we obtain

$$
B^{*} \gamma_{j}-H_{2}\left(y_{j}-y_{j-1}\right) \in \partial g\left(y_{j}\right) \quad \forall j \geq 1 .
$$

For every $k \geq 2$, using the previous inclusion for $j=k-1$ and $j=k$, it follows from the monotonicity of the subdifferential of $g$ that

$$
\left\langle B^{*}\left(\gamma_{k}-\gamma_{k-1}\right)-H_{2}\left(y_{k}-y_{k-1}\right)+H_{2}\left(y_{k-1}-y_{k-2}\right), y_{k}-y_{k-1}\right\rangle \geq 0,
$$

which, combined with (19), yields

$$
\left\langle B \Delta y_{k}, \Delta \gamma_{k}\right\rangle \geq\left\|\Delta y_{k}\right\|_{H_{2}}^{2}-\left\langle H_{2} \Delta y_{k-1}, \Delta y_{k}\right\rangle \quad \forall k \geq 2 .
$$

To conclude the proof, use the relation (4) with $Q=H_{2}$.

The following theorem shows that the generalized ADMM is an instance of the modified HPE framework. Let us consider the following quantity:

$$
\sigma_{\alpha}=\frac{1}{1+\alpha(2-\alpha)} .
$$

Note that $\sigma_{2}=1$, and for any $\alpha \in(0,2)$ we have $\sigma_{\alpha} \in(0,1)$.

Theorem 3.3. Let $\left\{\left(x_{k}, y_{k}, \gamma_{k}\right)\right\}$ be generated by the generalized ADMM and consider $\left\{\left(\Delta y_{k}, \tilde{\gamma}_{k}\right)\right\}$ and $\sigma_{\alpha}$ as in (19) and (28), respectively. Define

$$
z_{k-1}=\left(x_{k-1}, y_{k-1}, \gamma_{k-1}\right) \quad \tilde{z}_{k}=\left(x_{k}, y_{k}, \tilde{\gamma}_{k}\right), \quad \forall k \geq 1,
$$

and

$$
\eta_{0}=\frac{4(2-\alpha) \sigma_{\alpha}}{\alpha} d_{0}, \quad \eta_{k}=\frac{(2-\alpha) \sigma_{\alpha}}{\alpha}\left\|\Delta y_{k}\right\|_{H_{2}}^{2} \quad \forall k \geq 1,
$$

where $d_{0}$ is as in (18). Then, the sequence $\left\{\left(z_{k}, \tilde{z}_{k}, \eta_{k}\right)\right\}$ is an instance of the modified HPE framework applied for solving (13), where $\sigma:=\sigma_{\alpha}$ and $M$ is as in (17). 
Proof. The inclusion (7) follows from the last statement in Lemma 3.1. Let us now show that (8) holds. Using (19), (20) and (29), we obtain

$$
\begin{aligned}
\left\|\tilde{z}_{k}-z_{k}\right\|_{M}^{2} & =\frac{1}{\alpha \beta}\left\|\tilde{\gamma}_{k}-\gamma_{k}\right\|^{2}=\frac{1}{\alpha \beta}\left\|\frac{1}{\alpha}\left[(1-\alpha) \Delta \gamma_{k}+\beta B \Delta y_{k}\right]\right\|^{2} \\
& =\frac{1}{\alpha^{3} \beta}\left[(1-\alpha)^{2}\left\|\Delta \gamma_{k}\right\|^{2}+2(1-\alpha) \beta\left\langle B \Delta y_{k}, \Delta \gamma_{k}\right\rangle+\beta^{2}\left\|B \Delta y_{k}\right\|^{2}\right] .
\end{aligned}
$$

Also, (19) and (29) imply that

$$
\left\|\tilde{z}_{k}-z_{k-1}\right\|_{M}^{2}=\left\|\Delta x_{k}\right\|_{H_{1}}^{2}+\left\|\Delta y_{k}\right\|_{H_{2}}^{2}+\frac{\beta}{\alpha}\left\|B \Delta y_{k}\right\|^{2}+2 \frac{(1-\alpha)}{\alpha}\left\langle B \Delta y_{k}, \tilde{\gamma}_{k}-\gamma_{k-1}\right\rangle+\frac{1}{\alpha \beta}\left\|\tilde{\gamma}_{k}-\gamma_{k-1}\right\|^{2} .
$$

It follows from (20) that

$$
\begin{aligned}
\frac{1}{\alpha \beta}\left\|\tilde{\gamma}_{k}-\gamma_{k-1}\right\|^{2} & =\frac{1}{\alpha^{3} \beta}\left[\left\|\Delta \gamma_{k}\right\|^{2}+2 \beta\left\langle B \Delta y_{k}, \Delta \gamma_{k}\right\rangle+\beta^{2}\left\|B \Delta y_{k}\right\|^{2}\right], \\
2 \frac{(1-\alpha)}{\alpha}\left\langle B \Delta y_{k}, \tilde{\gamma}_{k}-\gamma_{k-1}\right\rangle & =2 \frac{(1-\alpha)}{\alpha^{2}}\left[\left\langle B \Delta y_{k}, \Delta \gamma_{k}\right\rangle+\beta\left\|B \Delta y_{k}\right\|^{2}\right]
\end{aligned}
$$

which, combined with (32), yields

$$
\begin{aligned}
\left\|\tilde{z}_{k}-z_{k-1}\right\|_{M}^{2}=\left\|\Delta x_{k}\right\|_{H_{1}}^{2} & +\left\|\Delta y_{k}\right\|_{H_{2}}^{2}+\left(\frac{\beta}{\alpha}+2 \frac{(1-\alpha) \beta}{\alpha^{2}}+\frac{\beta}{\alpha^{3}}\right)\left\|B \Delta y_{k}\right\|^{2} \\
& +2\left(\frac{(1-\alpha)}{\alpha^{2}}+\frac{1}{\alpha^{3}}\right)\left\langle B \Delta y_{k}, \Delta \gamma_{k}\right\rangle+\frac{1}{\alpha^{3} \beta}\left\|\Delta \gamma_{k}\right\|^{2} .
\end{aligned}
$$

Therefore, combining (31) and (33), it is easy to verify that

$$
\begin{aligned}
\sigma_{\alpha}\left\|\tilde{z}_{k}-z_{k-1}\right\|_{M}^{2} & -\left\|\tilde{z}_{k}-z_{k}\right\|_{M}^{2} \\
& =\sigma_{\alpha}\left\|\Delta x_{k}\right\|_{H_{1}}^{2}+\sigma_{\alpha}\left\|\Delta y_{k}\right\|_{H_{2}}^{2}+2 \frac{(2-\alpha) \sigma_{\alpha}}{\alpha}\left\langle B \Delta y_{k}, \Delta \gamma_{k}\right\rangle+\frac{(2-\alpha)^{2} \sigma_{\alpha}}{\alpha \beta}\left\|\Delta \gamma_{k}\right\|^{2} \\
& \geq 2 \frac{(2-\alpha) \sigma_{\alpha}}{\alpha}\left\langle B \Delta y_{k}, \Delta \gamma_{k}\right\rangle \geq \eta_{k}-\eta_{k-1} \quad \forall k \geq 1,
\end{aligned}
$$

where $\sigma_{\alpha}$ is as in (28), and the last inequality is due to (25) and (30). Therefore, (8) holds, and then we conclude that the sequence $\left\{\left(z_{k}, \tilde{z}_{k}, \eta_{k}\right)\right\}$ is an instance of the modified HPE framework.

\subsection{Iteration-complexity bounds for the generalized ADMM}

In this subsection, we study pointwise and ergodic iteration-complexity bounds for the generalized ADMM. We start by presenting a pointwise bound under the assumption that the relaxation parameter $\alpha$ belongs to $(0,2)$. Then, we consider an auxiliary result which is used to show that the sequence $\left\{\rho_{k}\right\}$, as defined in Theorem $\left[2.3\right.$ with $\left\{z_{k}\right\}$ and $\left\{\tilde{z}_{k}\right\}$ as in (29), is bounded even in the extreme case in which $\alpha=2$. This latter result is then used to present the ergodic bounds of the generalized ADMM for any $\alpha \in(0,2]$.

Theorem 3.4. (Pointwise convergence of the generalized ADMM) Let $\left\{\left(x_{k}, y_{k}, \gamma_{k}\right)\right\}$ be generated by the generalized $A D M M$ with $\alpha \in(0,2)$ and consider the sequence $\left\{\left(\Delta x_{k}, \Delta y_{k}, \Delta \gamma_{k}, \tilde{\gamma}_{k}\right)\right\}$ as in (19). Then, for every $k \geq 1$,

$$
0 \in M\left(\begin{array}{c}
\Delta x_{k} \\
\Delta y_{k} \\
\Delta \gamma_{k}
\end{array}\right)+\left(\begin{array}{c}
\partial f\left(x_{k}\right)-A^{*} \tilde{\gamma}_{k} \\
\partial g\left(y_{k}\right)-B^{*} \tilde{\gamma}_{k} \\
A x_{k}+B y_{k}-b
\end{array}\right)
$$


and there exists $i \leq k$ such that

$$
\left\|\left(\Delta x_{i}, \Delta y_{i}, \Delta \gamma_{i}\right)\right\|_{M} \leq \frac{1}{\sqrt{k}} \sqrt{\frac{2\left[\alpha\left(1+\sigma_{\alpha}\right)+8(2-\alpha) \sigma_{\alpha}\right] d_{0}}{\alpha\left(1-\sigma_{\alpha}\right)}}
$$

where $M, d_{0}$, and $\sigma_{\alpha}$ are as (17), (18) and (28), respectively.

Proof. Since $\sigma_{\alpha} \in(0,1)$ for any $\alpha \in(0,2)$ (see (28)), we obtain by combining Theorems 2.2 and 3.3 that (34) holds and there exists $i \leq k$ such that

$$
\left\|\left(\Delta x_{i}, \Delta y_{i}, \Delta \gamma_{i}\right)\right\|_{M} \leq \frac{1}{\sqrt{k}} \sqrt{\frac{2\left(1+\sigma_{\alpha}\right) d_{0}+4 \eta_{0}}{1-\sigma_{\alpha}}} .
$$

Hence, to conclude the proof use the definition of $\eta_{0}$ given in (30).

For a given tolerance $\varepsilon>0$, Theorem 3.4 implies that in at most $\mathcal{O}\left(1 / \varepsilon^{2}\right)$ iterations, the G-ADMM obtains an " $\varepsilon$-approximate" solution $(x, y, \gamma)$ and a residual $v$ of (3) satisfying

$$
M v \in T(x, y, \gamma), \quad\|v\|_{M} \leq \varepsilon
$$

where $T$ and $M$ are as (13) and (17), respectively.

Next we consider an auxiliary result which will be used to obtain ergodic iteration-complexity bounds for the generalized ADMM.

Lemma 3.5. Let $\left\{\left(x_{k}, y_{k}, \gamma_{k}\right)\right\}$ be generated by the generalized ADMM and consider $\left\{\left(\Delta x_{k}, \Delta y_{k}, \Delta \gamma_{k}, \tilde{\gamma}_{k}\right)\right\}$ as in (19). Then, the sequence $\left\{\rho_{k}\right\}$ given in (11) with $M$ and $\left\{\left(z_{k}, \tilde{z}_{k}\right)\right\}$ as in (17) and (29), respectively, satisfies

$$
\rho_{k} \leq \frac{4(1+2 \alpha)\left[\alpha+4(2-\alpha) \sigma_{\alpha}\right] d_{0}}{\alpha^{3}} \quad \forall k \geq 1,
$$

where $d_{0}$ is as in (18).

Proof. The same argument used to prove (32) and (33) yields, for every $k \geq 1$,

$$
\left\|\tilde{z}_{k}-z_{k-1}\right\|_{M}^{2}=\left\|\Delta x_{k}\right\|_{H_{1}}^{2}+\left\|\Delta y_{k}\right\|_{H_{2}}^{2}+\xi_{k}
$$

where

$$
\xi_{k}:=\frac{\beta}{\alpha^{3}}\left\|B \Delta y_{k}\right\|^{2}+\frac{2(1-\alpha)}{\alpha^{3}}\left\langle B \Delta y_{k}, \Delta \gamma_{k}\right\rangle+\frac{1}{\alpha^{3} \beta}\left\|\Delta \gamma_{k}\right\|^{2}+\frac{(2-\alpha)}{\alpha}\left[\frac{\beta}{\alpha}\left\|B \Delta y_{k}\right\|^{2}+\frac{2}{\alpha}\left\langle B \Delta y_{k}, \Delta \gamma_{k}\right\rangle\right] .
$$

Using the definitions of $M$ and $z_{k}$ given in (17) and (29), respectively, it follow that

$$
\begin{aligned}
\xi_{k} & \leq \frac{1}{\alpha^{2}}\left\|z_{k}-z_{k-1}\right\|_{M}^{2}+\frac{(2-\alpha)}{\alpha}\left[\frac{\beta}{\alpha}\left\|B \Delta y_{k}\right\|^{2}+\frac{2}{\alpha}\left\langle B \Delta y_{k}, \Delta \gamma_{k}\right\rangle\right] \\
& =\frac{1}{\alpha^{2}}\left\|z_{k}-z_{k-1}\right\|_{M}^{2}+\frac{(2-\alpha)}{\alpha}\left[\frac{\beta}{\alpha}\left\|B \Delta y_{k}\right\|^{2}+\frac{2(1-\alpha)}{\alpha}\left\langle B \Delta y_{k}, \Delta \gamma_{k}\right\rangle\right]+\frac{2(2-\alpha)}{\alpha}\left\langle B \Delta y_{k}, \Delta \gamma_{k}\right\rangle \\
& \leq \frac{1}{\alpha^{2}}\left\|z_{k}-z_{k-1}\right\|_{M}^{2}+\frac{(2-\alpha)}{\alpha}\left\|z_{k}-z_{k-1}\right\|_{M}^{2}+\frac{2(1-\alpha)}{\alpha}\left\langle B \Delta y_{k}, \Delta \gamma_{k}\right\rangle+\frac{2}{\alpha}\left\langle B \Delta y_{k}, \Delta \gamma_{k}\right\rangle \\
& \leq \frac{1+2 \alpha-\alpha^{2}}{\alpha^{2}}\left\|z_{k}-z_{k-1}\right\|_{M}^{2}+\frac{2(1-\alpha)}{\alpha}\left\langle B \Delta y_{k}, \Delta \gamma_{k}\right\rangle+\frac{\beta}{\alpha}\left\|B \Delta y_{k}\right\|^{2}+\frac{1}{\alpha \beta}\left\|\Delta \gamma_{k}\right\|^{2},
\end{aligned}
$$

where in the last two inequalities we used the fact that $\alpha \in(0,2]$ and (4) with $Q=I_{m}$, respectively. Combining (35), (36) and definitions of $M$ and $z_{k}$, we obtain, for every $k \geq 1$,

$$
\left\|\tilde{z}_{k}-z_{k-1}\right\|_{M}^{2} \leq \frac{1+2 \alpha-\alpha^{2}}{\alpha^{2}}\left\|z_{k}-z_{k-1}\right\|_{M}^{2}+\left\|z_{k}-z_{k-1}\right\|_{M}^{2}=\frac{1+2 \alpha}{\alpha^{2}}\left\|z_{k}-z_{k-1}\right\|_{M}^{2} .
$$


Now, letting $z^{*}:=\left(x^{*}, y^{*}, \gamma^{*}\right)$ be an arbitrary solution of (13), we obtain from the last inequality and (4) with $Q=M$ that

$$
\left\|\tilde{z}_{k}-z_{k-1}\right\|_{M}^{2} \leq \frac{2(1+2 \alpha)}{\alpha^{2}}\left[\left\|z^{*}-z_{k}\right\|_{M}^{2}+\left\|z^{*}-z_{k-1}\right\|_{M}^{2}\right] \quad \forall k \geq 1 .
$$

Since the generalized ADMM is an instance of the modified HPE framework with $\sigma:=\sigma_{\alpha}$ (see Theorem 3.3 and (28) $)$, it follows from the last inequality and Lemma A.1(b) that

$$
\left\|\tilde{z}_{k}-z_{k-1}\right\|_{M}^{2} \leq \frac{4(1+2 \alpha)}{\alpha^{2}}\left[\left\|z^{*}-z_{0}\right\|_{M}^{2}+\eta_{0}\right] \quad \forall k \geq 1 .
$$

Since $z^{*}$ is an arbitrary solution of (13), the result follows from the definition of $\rho_{k}, d_{0}$, and $\eta_{0}$ given in (11), (18) and (30), respectively.

Next result presents $\mathcal{O}(1 / k)$ convergence rate for the ergodic sequence associated to the generalized ADMM.

Theorem 3.6. (Ergodic convergence of the generalized ADMM) Let $\left\{\left(x_{k}, y_{k}, \gamma_{k}\right)\right\}$ be the sequence generated by the generalized ADMM and consider $\left\{\left(\Delta x_{k}, \Delta y_{k}, \Delta \gamma_{k}, \tilde{\gamma}_{k}\right)\right\}$ as in (19). Define the ergodic sequences as

$$
\begin{aligned}
\left(x_{k}^{a}, y_{k}^{a}, \gamma_{k}^{a}, \tilde{\gamma}_{k}^{a}\right) & =\frac{1}{k} \sum_{i=1}^{k}\left(x_{i}, y_{i}, \gamma_{i}, \tilde{\gamma}_{i}\right), \quad\left(r_{k, x}^{a}, r_{k, y}^{a}, r_{k, \gamma}^{a}\right)=\frac{1}{k} \sum_{i=1}^{k}\left(\Delta x_{i}, \Delta y_{i}, \Delta \gamma_{i}\right), \\
\varepsilon_{k, x}^{a} & =\frac{1}{k} \sum_{i=1}^{k}\left\langle H_{1} \Delta x_{i}-A^{*} \tilde{\gamma}_{i}, x_{k}^{a}-x_{i}\right\rangle, \\
\varepsilon_{k, y}^{a} & =\frac{1}{k} \sum_{i=1}^{k}\left\langle\left(H_{2}+\frac{\beta}{\alpha} B^{*} B\right) \Delta y_{i}+\frac{(1-\alpha)}{\alpha} B^{*} \Delta \gamma_{i}-B^{*} \tilde{\gamma}_{i}, y_{k}^{a}-y_{i}\right\rangle .
\end{aligned}
$$

Then, for every $k \geq 1$, there hold $\varepsilon_{k, x}^{a} \geq 0, \varepsilon_{k, y}^{a} \geq 0$, and

$$
\begin{gathered}
0 \in M\left(\begin{array}{c}
r_{k, x}^{a} \\
r_{k, y}^{a} \\
r_{k, \gamma}^{a}
\end{array}\right)+\left(\begin{array}{c}
\partial f_{\varepsilon_{k, x}^{a}}\left(x_{k}^{a}\right)-A^{*} \tilde{\gamma}_{k}^{a} \\
\partial g_{\varepsilon_{k, y}^{a}}\left(y_{k}^{a}\right)-B^{*} \tilde{\gamma}_{k}^{a} \\
A x_{k}^{a}+B y_{k}^{a}-b
\end{array}\right), \\
\left\|\left(r_{k, x}^{a}, r_{k, y}^{a}, r_{k, \gamma}^{a}\right)\right\|_{M} \leq \frac{2 \sqrt{c_{\alpha} d_{0}}}{k}, \quad \varepsilon_{k, x}^{a}+\varepsilon_{k, y}^{a} \leq \frac{\tilde{c}_{\alpha} d_{0}}{k},
\end{gathered}
$$

where

$$
c_{\alpha}:=\frac{\alpha+4(2-\alpha) \sigma_{\alpha}}{\alpha}, \quad \tilde{c}_{\alpha}:=\frac{3\left[3 \alpha^{2}+4(1+2 \alpha) \sigma_{\alpha}\right]\left[\alpha+4(2-\alpha) \sigma_{\alpha}\right]}{2 \alpha^{3}},
$$

and $M, d_{0}$, and $\sigma_{\alpha}$ are as in (17), (18), and (28), respectively.

Proof. It follows from Theorem 3.3 that the generalized ADMM is an instance of the modified HPE where $\left\{\left(z_{k}, \tilde{z}_{k}\right)\right\}$ is given by (29). Moreover, it is easy to see that the quantities $r_{k}^{a}$ and $\varepsilon_{k}^{a}$ given in (10) satisfy

$$
r_{k}^{a}=\left(r_{k, x}^{a}, r_{k, y}^{a}, r_{k, \gamma}^{a}\right), \quad \varepsilon_{k}^{a}=\frac{1}{k} \sum_{i=1}^{k}\left[\left\langle M\left(\begin{array}{c}
\Delta x_{i} \\
\Delta y_{i} \\
\Delta \gamma_{i}
\end{array}\right),\left(x_{k}^{a}-x_{i}, y_{k}^{a}-y_{i}, \tilde{\gamma}_{k}^{a}-\tilde{\gamma}_{i}\right)\right\rangle\right] .
$$

Hence, from Theorems 2.3 and definition of $\eta_{0}$ in (30), we have

$$
\left\|r_{k}^{a}\right\|_{M} \leq \frac{2 \sqrt{\left(\alpha+4(2-\alpha) \sigma_{\alpha}\right) d_{0}}}{k \sqrt{\alpha}}, \quad \varepsilon_{k}^{a} \leq \frac{3\left[3 \alpha^{2}+4(1+2 \alpha) \sigma_{\alpha}\right]\left[\alpha+4(2-\alpha) \sigma_{\alpha}\right] d_{0}}{2 \alpha^{3} k},
$$


where in the last inequality we also used Lemma 3.5. Now, we claim that $\varepsilon_{k}^{a}=\varepsilon_{k, x}^{a}+\varepsilon_{k, y}^{a}$. Using this claim, (41) follows immediately from (42) and (44). Hence, to conclude the proof of the theorem, it just remains to prove the above claim. To this end, note that (38) and (39) yield

$$
\begin{aligned}
\varepsilon_{k, x}^{a}+\varepsilon_{k, y}^{a} & =\frac{1}{k} \sum_{i=1}^{k}\left[\left\langle H_{1} \Delta x_{i}, x_{k}^{a}-x_{i}\right\rangle+\left\langle\left(H_{2}+\frac{\beta}{\alpha} B^{*} B\right) \Delta y_{i}+\frac{(1-\alpha)}{\alpha} B^{*} \Delta \gamma_{i}, y_{k}^{a}-y_{i}\right\rangle\right] \\
& \left.+\frac{1}{k} \sum_{i=1}^{k}\left\langle A\left(x_{k}^{a}-x_{i}\right)+B\left(y_{k}^{a}-y_{i}\right),-\tilde{\gamma}_{i}\right\rangle\right] .
\end{aligned}
$$

On the other hand, from (37), we obtain

$$
\begin{aligned}
\frac{1}{k} \sum_{i=1}^{k}\left\langle A\left(x_{k}^{a}-x_{i}\right)+B\left(y_{k}^{a}-y_{i}\right),-\tilde{\gamma}_{i}\right\rangle & =\frac{1}{k} \sum_{i=1}^{k}\left\langle A x_{k}^{a}+B y_{k}^{a}-b-\left(A x_{i}+B y_{i}-b\right), \tilde{\gamma}_{k}^{a}-\tilde{\gamma}_{i}\right\rangle \\
& =\frac{1}{k} \sum_{i=1}^{k}\left\langle-\left(A x_{i}+B y_{i}-b\right), \tilde{\gamma}_{k}^{a}-\tilde{\gamma}_{i}\right\rangle \\
& =\frac{1}{k} \sum_{i=1}^{k}\left\langle\frac{(1-\alpha)}{\alpha} B \Delta y_{i}+\frac{1}{\alpha \beta} \Delta \gamma_{i}, \tilde{\gamma}_{k}^{a}-\tilde{\gamma}_{i}\right\rangle
\end{aligned}
$$

where the last equality is due to (23). Hence, the claim follows by combining (45), and the definitions of $M$ and $\varepsilon_{k}^{a}$ in (17) and (43), respectively.

For a given tolerance $\varepsilon>0$, Theorem 3.6 implies that in at most $\mathcal{O}(1 / \varepsilon)$ iterations of the G-ADMM, we obtain an " $\varepsilon$-approximate" solution $(\hat{x}, \hat{y}, \hat{\gamma})$ and a residual $\hat{v}=\left(\hat{v}_{1}, \hat{v}_{2}, \hat{v}_{3}\right)$ of (3) satisfying

$$
\hat{v}_{1} \in \partial_{\varepsilon_{1}} f(\hat{x})-A^{*} \hat{\gamma}, \quad \hat{v}_{2} \in \partial_{\varepsilon_{2}} g(\hat{y})-B^{*} \hat{\gamma}, \quad \hat{v}_{3}=A \hat{x}+B \hat{y}-b, \quad\|\hat{v}\|_{M}^{*} \leq \varepsilon, \quad \varepsilon_{1}+\varepsilon_{2} \leq \varepsilon,
$$

where $\|\cdot\|_{M}^{*}$ is a dual norm (seminorm) associated to $M$.

\section{A Appendix (Proofs of Theorems 2.2 and 2.3}

The main goal in this section is to present the proofs of Theorems 2.2 and 2.3 . Toward this goal, we first consider a technical lemma.

Lemma A.1. Let $\left\{\left(z_{k}, \tilde{z}_{k}, \eta_{k}\right)\right\}$ be the sequence generated by the modified HPE framework. For every $k \geq 1$, the following statements hold:

(a) for every $z \in \mathcal{Z}$, we have

$$
\left\|z-z_{k}\right\|_{M}^{2}+\eta_{k} \leq(\sigma-1)\left\|\tilde{z}_{k}-z_{k-1}\right\|_{M}^{2}+\left\|z-z_{k-1}\right\|_{M}^{2}+2\left\langle M\left(z_{k-1}-z_{k}\right), z-\tilde{z}_{k}\right\rangle+\eta_{k-1} ;
$$

(b) for every $z^{*} \in T^{-1}(0)$, we have

$$
\left\|z^{*}-z_{k}\right\|_{M}^{2}+\eta_{k} \leq(\sigma-1)\left\|\tilde{z}_{k}-z_{k-1}\right\|_{M}^{2}+\left\|z^{*}-z_{k-1}\right\|_{M}^{2}+\eta_{k-1} \leq\left\|z^{*}-z_{k-1}\right\|_{M}^{2}+\eta_{k-1} .
$$

Proof. (a) Note that, for every $z \in \mathcal{Z}$,

$$
\begin{aligned}
\left\|z-z_{k}\right\|_{M}^{2}-\left\|z-z_{k-1}\right\|_{M}^{2} & =\left\|\left(z-\tilde{z}_{k}\right)+\left(\tilde{z}_{k}-z_{k}\right)\right\|_{M}^{2}-\left\|\left(z-\tilde{z}_{k}\right)+\left(\tilde{z}_{k}-z_{k-1}\right)\right\|_{M}^{2} \\
& =\left\|\tilde{z}_{k}-z_{k}\right\|_{M}^{2}-\left\|\tilde{z}_{k}-z_{k-1}\right\|_{M}^{2}+2\left\langle M\left(z_{k-1}-z_{k}\right), z-\tilde{z}_{k}\right\rangle,
\end{aligned}
$$

which, combined with (8), proves the desired inequaliy.

(b) Since $M\left(z_{k-1}-z_{k}\right) \in T\left(\tilde{z}_{k}\right)$ and $0 \in T\left(z^{*}\right)$, we have $\left\langle M\left(z_{k-1}-z_{k}\right), \tilde{z}_{k}-z^{*}\right\rangle \geq 0$. Hence, the first inequality in (b) follows from (a) with $z=z^{*}$. Now, the second inequality in (b) follows from the fact that $\sigma \leq 1$ 
Proof of Theorem 2.2; The inclusion $0 \in M\left(z_{k}-z_{k-1}\right)+T\left(\tilde{z}_{k}\right)$ holds due to (7). It follows from (4) with $Q=M$ that, for every $j \geq 1$,

$$
\left.\left\|z_{j}-z_{j-1}\right\|_{M}^{2} \leq 2\left(\| \tilde{z}_{j}-z_{j-1}\right)\left\|_{M}^{2}+\right\| \tilde{z}_{j}-z_{j} \|_{M}^{2}\right) \leq 2(1+\sigma)\left\|\tilde{z}_{j}-z_{j-1}\right\|_{M}^{2}+2\left(\eta_{j-1}-\eta_{j}\right)
$$

where the last inequality is due to (8). Now, if $z^{*} \in T^{-1}(0)$, we obtain from Lemma A.1(b)

$$
(1-\sigma)\left\|\tilde{z}_{j}-z_{j-1}\right\|_{M}^{2} \leq\left\|z^{*}-z_{j-1}\right\|_{M}^{2}-\left\|z^{*}-z_{j}\right\|_{M}^{2}+\eta_{j-1}-\eta_{j}, \quad \forall j \geq 1 .
$$

Combining the last two inequality, we get

$$
\begin{aligned}
(1-\sigma) \sum_{j=1}^{k}\left\|z_{j}-z_{j-1}\right\|_{M}^{2} & \leq 2(1+\sigma)\left(\left\|z^{*}-z_{0}\right\|_{M}^{2}-\left\|z^{*}-z_{k}\right\|_{M}^{2}+\eta_{0}-\eta_{k}\right)+2(1-\sigma)\left(\eta_{0}-\eta_{k}\right) \\
& \leq 2(1+\sigma)\left\|z^{*}-z_{0}\right\|_{M}^{2}+4 \eta_{0} .
\end{aligned}
$$

Hence, as $\sigma<1$, we obtain

$$
\min _{i=1, \ldots, k}\left\|z_{i}-z_{i-1}\right\|_{M}^{2} \leq \frac{1}{k(1-\sigma)}\left(2(1+\sigma)\left\|z^{*}-z_{0}\right\|_{M}^{2}+4 \eta_{0}\right) .
$$

Therefore, the desired inequality follows from the latter inequality and the definition of $d_{0}$ in (9).

Proof of Theorem 2.3: The inclusion $0 \in M r_{k}^{a}+T^{\left[\varepsilon_{k}^{a}\right]}\left(\tilde{z}_{k}^{a}\right)$ follows from (7), (10), and Theorems 2.1(a). Using (10), it is easy see that for any $z^{*} \in T^{-1}(0)$

$$
k r_{k}^{a}=z_{k}-z_{0}=\left(z^{*}-z_{0}\right)+\left(z_{k}-z^{*}\right) .
$$

Hence, from inequality (4) with $Q=M$ and Lemma A.1(b), we have

$$
k^{2}\left\|r_{k}^{a}\right\|_{M}^{2} \leq 2\left(\left\|z^{*}-z_{0}\right\|_{M}^{2}+\left\|z^{*}-z_{k}\right\|_{M}^{2}\right) \leq 4\left(\left\|z^{*}-z_{0}\right\|_{M}^{2}+\eta_{0}\right) .
$$

Combining the above inequality with definition of $d_{0}$, we obtain the bound on $\left\|r_{k}^{a}\right\|_{M}$. Let us now to prove the bound on $\varepsilon_{k}^{a}$. From Lemma A.1(a), we have

$$
2 \sum_{i=1}^{k}\left\langle M\left(z_{i}-z_{i-1}\right), z-\tilde{z}_{i}\right\rangle \leq\left\|z-z_{0}\right\|_{M}^{2}-\left\|z-z_{k}\right\|_{M}^{2}+\eta_{0}-\eta_{k} \leq\left\|z-z_{0}\right\|_{M}^{2}+\eta_{0},
$$

for every $z \in \mathcal{Z}$. Letting $z=\tilde{z}_{k}^{a}$ and using (10), we get

$$
2 k \varepsilon_{k}^{a} \leq\left\|\tilde{z}_{k}^{a}-z_{0}\right\|_{M}^{2}+\eta_{0} \leq \frac{1}{k} \sum_{i=1}^{k}\left\|\tilde{z}_{i}-z_{0}\right\|_{M}^{2}+\eta_{0} \leq \max _{i=1, \ldots, k}\left\|\tilde{z}_{i}-z_{0}\right\|_{M}^{2}+\eta_{0}
$$

where the second inequality is due to convexity of the function $\|\cdot\|_{M}^{2}$, which also implies that, for every $i \geq 1$ and $z^{*} \in T^{-1}(0)$,

$$
\left\|\tilde{z}_{i}-z_{0}\right\|_{M}^{2} \leq 3\left[\left\|\tilde{z}_{i}-z_{i}\right\|_{M}^{2}+\left\|z^{*}-z_{i}\right\|_{M}^{2}+\left\|z^{*}-z_{0}\right\|_{M}^{2}\right] .
$$

Hence, using (8) and twice Lemma A.1(b), it follows, for every $i \geq 1$ and $z^{*} \in T^{-1}(0)$, that

$$
\begin{aligned}
\left\|\tilde{z}_{i}-z_{0}\right\|_{M}^{2} & \leq 3\left[\sigma\left\|\tilde{z}_{i}-z_{i-1}\right\|_{M}^{2}+\eta_{i-1}+\left\|z^{*}-z_{i-1}\right\|_{M}^{2}+\eta_{i-1}+\left\|z^{*}-z_{0}\right\|_{M}^{2}\right] \\
& \leq 3\left[\sigma\left\|\tilde{z}_{i}-z_{i-1}\right\|_{M}^{2}+2\left(\left\|z^{*}-z_{i-1}\right\|_{M}^{2}+\eta_{i-1}\right)+\left\|z^{*}-z_{0}\right\|_{M}^{2}\right] \\
& \leq 3\left[\sigma\left\|\tilde{z}_{i}-z_{i-1}\right\|_{M}^{2}+3\left\|z^{*}-z_{0}\right\|_{M}^{2}+2 \eta_{0}\right]
\end{aligned}
$$


which, combined with (46) and definitions of $\rho_{k}$ in (11), yields

$$
2 k \varepsilon_{k}^{a} \leq 3\left[3\left\|z^{*}-z_{0}\right\|_{M}^{2}+\sigma \rho_{k}\right]+7 \eta_{0} \leq 3\left[3\left(\left\|z^{*}-z_{0}\right\|_{M}^{2}+\eta_{0}\right)+\sigma \rho_{k}\right] .
$$

Thus, the bound on $\varepsilon_{k}^{a}$ now follows from the definition of the $d_{0}$ in (9).

It remains to prove the second part of the theorem.

(a) if $\sigma<1$, then it follows from Lemma A.1(b), for every $i \geq 1$ and $z^{*} \in T^{-1}(0)$, that

$$
(1-\sigma)\left\|\tilde{z}_{i}-z_{i-1}\right\|_{M}^{2} \leq\left\|z^{*}-z_{i-1}\right\|_{M}^{2}+\eta_{i-1} \leq\left\|z^{*}-z_{0}\right\|_{M}^{2}+\eta_{0}
$$

Hence, in view of definitions of $\rho_{k}$ and $d_{0}$, we obtain (12).

(b) If Dom $T$ is bounded, then it follows from inequality (4) with $Q=M$, and Lemma A.1 (b), for every $i \geq 1$ and $z^{*} \in T^{-1}(0)$, that

$$
\left\|\tilde{z}_{i}-z_{i-1}\right\|_{M}^{2} \leq 2\left[\left\|z^{*}-z_{i-1}\right\|_{M}^{2}+\left\|\tilde{z}_{i}-z^{*}\right\|_{M}^{2}\right] \leq 2\left[\left\|z^{*}-z_{0}\right\|_{M}^{2}+\eta_{0}+D\right]
$$

which, combined with definitions of $\rho_{k}$ and $d_{0}$, proves the desired result.

\section{References}

[1] D. P. Bertsekas. Constrained optimization and Lagrange multiplier methods. Academic Press, New York, 1982.

[2] S. Boyd, N. Parikh, E. Chu, B. Peleato, and J. Eckstein. Distributed optimization and statistical learning via the alternating direction method of multipliers. Found. Trends Mach. Learn., 3(1):1-122, 2011.

[3] R. S. Burachik, A. N. Iusem, and B. F. Svaiter. Enlargement of monotone operators with applications to variational inequalities. Set-Valued Anal., 5(2):159-180, 1997.

[4] R. S. Burachik, C. A. Sagastizábal, and B. F. Svaiter. $\epsilon$-enlargements of maximal monotone operators: theory and applications. In Reformulation: nonsmooth, piecewise smooth, semismooth and smoothing methods (Lausanne, 1997), volume 22 of Appl. Optim., pages 25-43. Kluwer Acad. Publ., Dordrecht, 1999 .

[5] A. Chambolle and T. Pock. A first-order primal-dual algorithm for convex problems with applications to imaging. J. Math. Imaging Vis., 40(1):120-145, 2011.

[6] E. Corman and X. Yuan. A generalized proximal point algorithm and its convergence rate. SIAM Journal on Optimization, 24(4):1614-1638, 2014.

[7] Y. Cui, X. Li, D. Sun, and K. C. Toh. On the convergence properties of a majorized ADMM for linearly constrained convex optimization problems with coupled objective functions. J. Optim. Theory Appl., $169(3): 1013-1041,2016$.

[8] W. Deng and W. Yin. On the global and linear convergence of the generalized alternating direction method of multipliers. J. Sci. Comput., pages 1-28, 2015.

[9] J. Eckstein. Parallel alternating direction multiplier decomposition of convex programs. J. Optim. Theory Appl., 80(1):39-62, 1994.

[10] J. Eckstein. Some saddle-function splitting methods for convex programming. Optim. Method Softw., 4(1):75-83, 1994.

[11] J. Eckstein and D. P. Bertsekas. On the Douglas-Rachford splitting method and the proximal point algorithm for maximal monotone operators. Math. Programming, 55(3, Ser. A):293-318, 1992. 
[12] E. X. Fang, B. He, H. Liu, and X. Yuan. Generalized alternating direction method of multipliers: new theoretical insights and applications. Math. Prog. Comp., 7(2):149-187, 2015.

[13] D. Gabay and B. Mercier. A dual algorithm for the solution of nonlinear variational problems via finite element approximation. Comput. Math. Appl., 2:17-40, 1976.

[14] R. Glowinski. Numerical Methods for Nonlinear Variational Problems. Springer Series in Computational Physics. Springer-Verlag, 1984.

[15] R. Glowinski and A. Marroco. Sur l'approximation, par éléments finis d'ordre un, et la résolution, par penalisation-dualité, d'une classe de problèmes de Dirichlet non linéaires. 1975.

[16] M. L. N. Gonçalves, J. G. Melo, and M. M. Alves. Pointwise and ergodic convergence rates of a variable metric proximal ADMM. arXiv preprint arXiv:1702.06626, 2017.

[17] M. L. N. Gonçalves, J. G. Melo, and R. D. C. Monteiro. Extending the ergodic convergence rate of the proximal ADMM. arXiv preprint arXiv:1611.02903, 2016.

[18] M. L. N. Gonçalves, J. G. Melo, and R. D. C. Monteiro. Improved pointwise iteration-complexity of a regularized ADMM and of a regularized non-euclidean HPE framework. SIAM Journal on Optimization, 27(1):379-407, 2017.

[19] Y. Gu, B. Jiang, and H. Deren. A semi-proximal-based strictly contractive Peaceman-Rachford splitting method. Avaliable on http://www.arxiv.org.

[20] W. W. Hager, M. Yashtini, and H. Zhang. An $O(1 / k)$ convergence rate for the variable stepsize Bregman operator splitting algorithm. SIAM J. Numer. Anal., 54(3):1535-1556, 2016.

[21] B. He and X. Yuan. On the $\mathcal{O}(1 / n)$ convergence rate of the Douglas-Rachford alternating direction method. SIAM Journal on Numer. Anal., 50(2):700-709, 2012.

[22] B. He and X. Yuan. On non-ergodic convergence rate of Douglas-Rachford alternating direction method of multipliers. Numer. Math., 130(3):567-577, 2015.

[23] T. Lin, S. Ma, and S. Zhang. An extragradient-based alternating direction method for convex minimization. Found. Comput. Math., pages 1-25, 2015.

[24] R. D. C. Monteiro and B. F. Svaiter. On the complexity of the hybrid proximal extragradient method for the iterates and the ergodic mean. SIAM J. Optim., 20(6):2755-2787, 2010.

[25] R. D. C. Monteiro and B. F Svaiter. Iteration-complexity of block-decomposition algorithms and the alternating direction method of multipliers. SIAM J. Optim., 23(1):475-507, 2013.

[26] R. Nishihara, L. Lessard, B. Recht, A. Packard, and M. I. Jordan. A general analysis of the convergence of ADMM. In ICML, pages 343-352, 2015.

[27] Y. Ouyang, Y. Chen, G. Lan, and E. Pasiliao Jr. An accelerated linearized alternating direction method of multipliers. SIAM J. Imaging Sci., 8(1):644-681, 2015.

[28] R. T. Rockafellar. On the maximal monotonicity of subdifferential mappings. Pacific J. Math., 33:209216, 1970.

[29] M. V. Solodov and B. F. Svaiter. A hybrid approximate extragradient-proximal point algorithm using the enlargement of a maximal monotone operator. Set-Valued Anal., 7(4):323-345, 1999.

[30] H. Sun. Analysis of fully preconditioned ADMM with relaxation in hilbert spaces. arXiv preprint arXiv:1611.04801, 2016. 
[31] M. Tao and X. Yuan. On the optimal linear convergence rate of a generalized proximal point algorithm. arXiv preprint arXiv:1605.05474, 2016.

[32] X. Wang and X Yuan. The linearized alternating direction method of multipliers for dantzig selector. SIAM J. Sci. Comput., 34(5):2792-2811, 2012.

[33] J. Yang and X. Yuan. Linearized augmented Lagrangian and alternating direction methods for nuclear norm minimization. Math. Comput., 82(281):301-329, 2013. 